\title{
Spectral Gain Characteristics Induced by 980 nm Pumping Band in a Gain-flattened EDFA ${ }^{1}$
}

\author{
A. R. Sarmani ${ }^{a}$, S.-J Sheih ${ }^{b}$, F. R. Mahamd Adikan ${ }^{c}$, and M. A. Mahdi ${ }^{a}$ * \\ ${ }^{a}$ Wireless and Photonics Networks Research Center, Faculty of Engineering, Universiti Putra Malaysia, \\ 43400 UPM Serdang, Selangor, Malaysia \\ ${ }^{b}$ Taiwan International Securities Group, 33F, No. 97, Tun-Hwa S. Rd., Sec.2, Taipei 106, Taiwan \\ ${ }^{c}$ Department of Electrical Engineering, Faculty of Engineering, Universiti Malaya, \\ 50603 Kuala Lumpur, Malaysia \\ *e-mail:mdadzir@eng.upm.edu.my, adzir@.ieee.org \\ Received March 16, 2010; in final form, April 9, 2010; published online August 2, 2010
}

\begin{abstract}
We demonstrate the effect of inversion population ratio mediated by $980 \mathrm{~nm}$ pumping band on the gain spectra of a gain-flattened Erbium-doped fiber amplifier. The gain equalizing filter was designed at $977 \mathrm{~nm}$ and the amplifier was built in the four-stage arrangement. In the pumping structure, the $1480 \mathrm{~nm}$ lasers were maintained in the final gain block while the $980 \mathrm{~nm}$ band lasers were utilized in the first three blocks. These laser sources were arranged at several combinations of 977 and $980 \mathrm{~nm}$ wavelengths. Within these $3 \mathrm{~nm}$ spectrum, an absorption cross-section difference around $0.32 \times 10^{-25} \mathrm{~m}^{2}$ contributed to a dispensable gain variation up to $3-\mathrm{dB}$. These inequalities were the results of variations in population inversion induced by the pump wavelength discrepancy. The findings show the importance of designing a filter at the specific absorption wavelength to allow the operation of consistent gain level.
\end{abstract}

DOI: $10.1134 / \mathrm{S} 1054660 \mathrm{X} 10170135$

\section{INTRODUCTION}

Erbium-doped fiber amplifiers (EDFAs) [1-4] that operate in the telecommunication window around $1.5 \mu \mathrm{m}$ have wide applications for wavelength division multiplexing (WDM) systems. During transmissions, the uniformity of gain amplitude and acceptable optical-to-signal noise ratio (OSNR) [5] must be maintained. These are realized by incorporating an optical feedback technique [6], a double-pass setup [7], or a gain equalization filter (GEF) into an EDFA module [8]. Previously reported works had demonstrated a wavelength independent gain operation from EDFA's with gain discrepancies of just $\pm 0.5 \mathrm{~dB}$ and less over a bandwidth around $30 \mathrm{~nm}$ in the C-band domain [9, 10]. In addition, an EDFA was also developed successfully in the extended L-band by utilizing the effect of stimulated Raman scattering in the pumping scheme [11] or a novel low noise silicatefiber [12].

The output profile of an amplifier is a function of emitting wavelengths. By modifying the inversion population ratio $\bar{n}_{2}$, the spectral gain dependency [13] are induced in the EDFA. The coupling of gain feature and amplitude to the absorption and emission properties of the fiber medium is described as "dynamic gain tilt" (DGT) [14]. The suppression of DGT can be done by exploiting the inversion factor $\bar{n}_{2}$, that is influenced by the pump wavelength. When designing

\footnotetext{
${ }^{1}$ The article is published in the original.
}

an EDFA that can achieve similar gain level, these factors must be taken into consideration. If $\bar{n}_{2}$ is not equal between the designed and experimental values, a substantial fluctuation in the emitting spectra can be observed.

In this letter, we investigate the pump wavelength dependency effect to the inversion population factor that influences the output gain profile of the amplifier. Various combinations of $980 \mathrm{~nm}$ pumping band were utilized to conclude the observations.

\section{EXPERIMENTAL SETUP}

The variable gain-flattened EDFA module that incorporated four gain blocks (GB) is presented in Fig. 1. It was based on an Erbium-doped fiber (EDF) that had a bandwidth of $35 \mathrm{~nm}$ for the wavelengths between 1529 to $1564 \mathrm{~nm}$. The absorption coefficient of the fiber was $5.9 \mathrm{~dB} / \mathrm{m}$ at $1530 \mathrm{~nm}$, the numerical aperture was 0.22 and the cutoff pump wavelength was $910 \mathrm{~nm}$. The EDF length distributions were 7, 9, 7, and $19 \mathrm{~m}$ for GB\#1 to GB\#4, consecutively. The pump sources $\mathrm{P} 1$ and $\mathrm{P} 2$ for $\mathrm{GB} \# 1-\mathrm{GB} \# 3$ were provided by $980 \mathrm{~nm}$ band laser diodes and for GB\#4, $1480 \mathrm{~nm}$ laser diodes were implemented as P3 and P4. Since GB\#1 had power restrictions, a 3-dB coupler was deployed to divide the pump powers equivalently to $\mathrm{GB} \# 1$ and GB\#3. In contrast, the GB\#2 was especially pumped by a $980 \mathrm{~nm}$ laser diode to maximize the signal gain in order to compensate the loss introduced by the dispersion compensating module (DCM). 


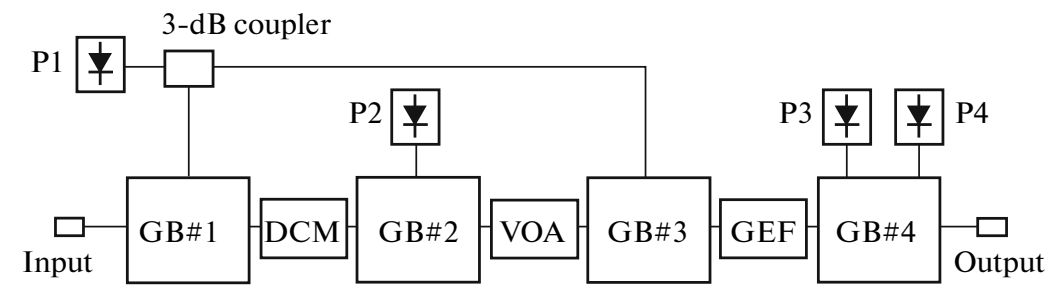

Fig. 1. Schematic diagram of four-stages EDFA with intra-stage elements of DCM, VOA, and GEF.

Maximum output powers up to $200 \mathrm{~mW}$ were produced from all the pump sources employed in this experiment.

The DCM was used to mitigate the problem of fiber dispersion and it introduced a maximum loss of $10 \mathrm{~dB}$. The output power from GB\#1 was set at $0.4 \mathrm{dBm} /$ channel that resulted in the total output power of $16 \mathrm{dBm}$ by considering the utilization of 40 channels (100 GHz and $0.8 \mathrm{~nm}$ spacing) in the transmission systems. This was satisfied to diminish the nonlinear effects in the dispersive component that could deteriorate the amplifier performance.

In the middle of EDFA structure, a variable optical attenuator (VOA) was incorporated to control the operating gain of EDFA from 15 to $30 \mathrm{~dB}$. A gain equalizing filter (GEF) was placed in between the last two gain blocks. The GEF was designed when pump sources P1 and P2 were fixed at $977 \mathrm{~nm}$ but during implementation, these were arranged in three different combinations. The first arrangement included both $980 \mathrm{~nm}$ laser diodes and the second included both $977 \mathrm{~nm}$ lasers as pump sources. In the third configuration, a $977 \mathrm{~nm}$ pump laser was employed for P1 and a $980 \mathrm{~nm}$ pump laser was used for P2. However in the whole assessment, P3 and P4 were retained at $1480 \mathrm{~nm}$ wavelength.

\section{RESULTS AND DISCUSSIONS}

Before performing any investigation on this set-up, analysis on the laser gain $G\left(\lambda_{s}\right)$ that is based on Giles model need to be done first. At the signal wavelength $\lambda_{s}$, this homogenous approximation is expressed as $[14,15]$;

$$
G\left(\lambda_{s}\right)={ }_{10 \log _{10}} \Gamma L\left(\bar{N}_{2} \sigma_{e}^{\lambda_{s}}-\bar{N}_{1} \sigma_{a}^{\lambda_{s}}\right),
$$

where $\Gamma$ is the overlap factor between the optical mode and erbium-doped core, $L$ is the length of the gain medium, $\bar{N}_{1}$ and $\bar{N}_{2}$ are average population densities of the ground state and excited level, correspondingly. Other parameters that are composed of $\sigma_{e}^{\lambda_{s}}$ and $\sigma_{a}^{\lambda_{s}}$, are emission and absorption cross-sections at the signal wavelength.

The total population of Erbium ions in the host medium $N_{\text {tot }}$, includes the average populations in both energy levels where $N_{\text {tot }}=\bar{N}_{1}+\bar{N}_{2}$. By introducing an inversion population ratio at the excited level $\bar{n}_{2}=$ $\bar{N}_{2} / N_{\text {tot }}$, Eq. (1) can be transposed into;

$$
G\left(\lambda_{s}\right)=10 \log _{10} \Gamma L N_{\text {tot }}\left[\bar{n}_{2}\left(\sigma_{e}^{\lambda_{s}}+\sigma_{a}^{\lambda_{s}}\right)-\sigma_{a}^{\lambda_{s}}\right],
$$

where all parameters that contribute to the function in Eq. (2) such as the length and spectroscopic properties of the fiber have been maintained consistently, changing $\bar{n}_{2}$ is the only way available for varying the gain value.

The influence of different pump wavelengths $\lambda_{p}$ on $\bar{n}_{2}$ that affects the laser gain, can be estimated from spectroscopic properties of the active ions. In Fig. 2, the absorption spectrum of an erbium-doped fiber in the near infrared wavelengths is illustrated. The absorption cross-sections $\left(\sigma_{a}^{\lambda_{p}}\right)$ are finite while the emission cross-sections are zero within this region. In addition, a sharp slope can be seen for wavelengths that are shorter and longer than the peak wavelength absorption at $978 \mathrm{~nm}$. The $\sigma_{a}^{\lambda_{p}}$ at $980 \mathrm{~nm}$ is $1.96 \times$ $10^{-25} \mathrm{~m}^{2}$ and at $977 \mathrm{~nm}$, it is $2.28 \times 10^{-25} \mathrm{~m}^{2}$. As $\bar{n}_{2}$ is proportional to $\sigma_{a}^{\lambda_{p}}, \bar{n}_{2}$ at the designed wavelength of $977 \mathrm{~nm}$ is not equivalent compared to that at $980 \mathrm{~nm}$. This effect can be observed by evaluating the results obtained in this assessment.

During operation, the input power was arranged at $-7 \mathrm{dBm}$ and the pump lasers were varied to maintain the composite output power to be at $23 \mathrm{dBm}$. In this case, the VOA was set to $0 \mathrm{~dB}$ to produce gain at $30 \mathrm{~dB}$. The characterization of EDFA module could be realized by deploying 40 channels simultaneously for a wavelength range from 1530 to $1562 \mathrm{~nm}$. The measurement at the output port was performed by using an optical spectrum analyzer where the results are demonstrated in Figs. 3 and 4.

From Fig. 3 where the set-up implemented two $980 \mathrm{~nm}$ pump lasers (see Fig. 1), a distinct fluctuation of power in $\mathrm{dBm}$ was observed where the discrepancy between its maximum to minimum was $3 \mathrm{~dB}$. When the amplifier gain saturated, the gain compression was higher at shorter wavelengths compared to that at 


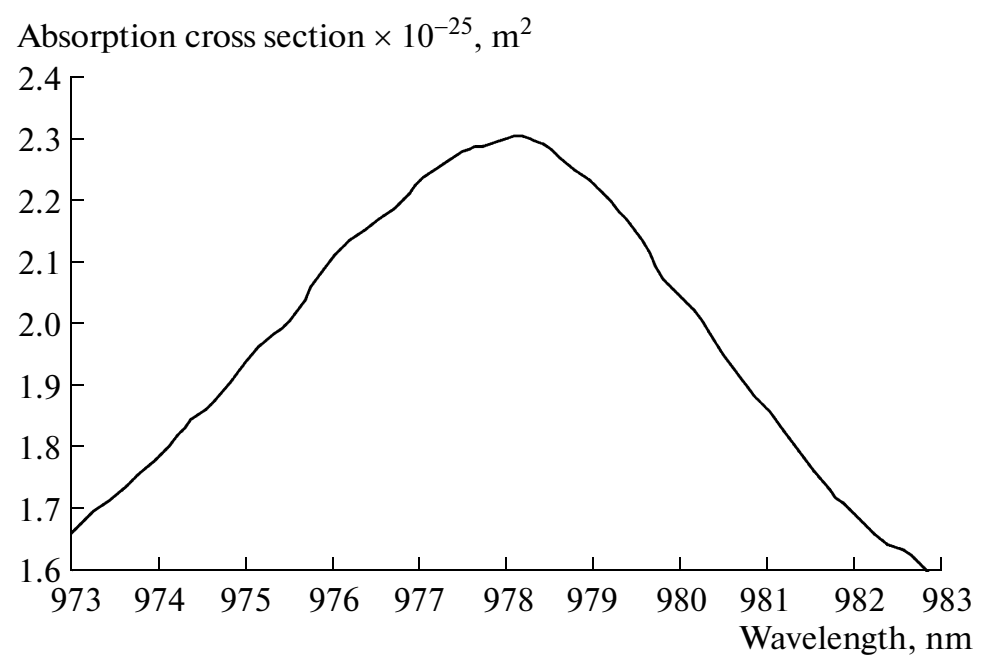

Fig. 2. Absorption cross-sections for the erbium-doped fiber used in the experiment.

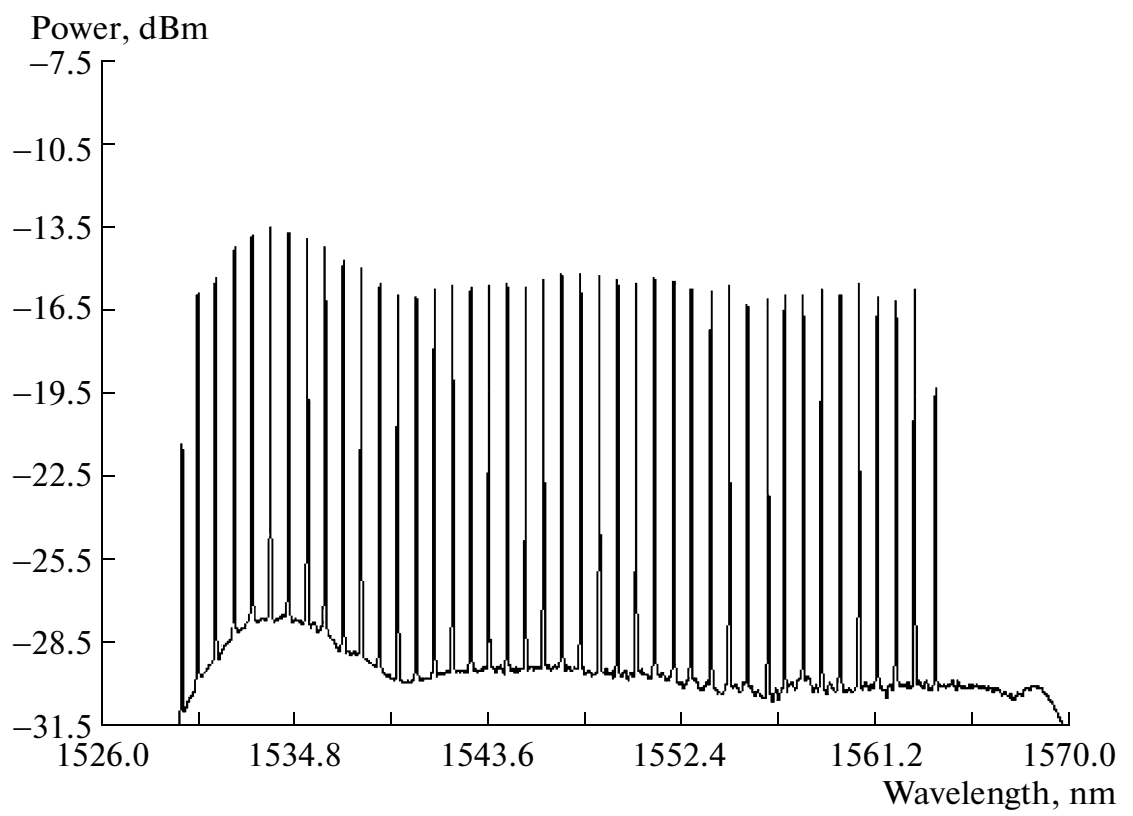

Fig. 3. Gain spectrum in the C-band (Gain $=30 \mathrm{~dB}$ ) for a combination of two $980 \mathrm{~nm}$ pump lasers.

longer wavelengths. This resulted in a "hump" in the wavelength domain between 1529.4 to $1538.6 \mathrm{~nm}$ that was caused by "spectral gain dependency" $[13,16]$. As DGT was initiated because $\Delta G\left(\lambda_{s}\right) / \bar{n}_{2} \propto \Gamma\left(\sigma_{e}^{\lambda_{s}}+\right.$ $\left.\sigma_{a}^{\lambda_{s}}\right)$, this area also implied the highest change in the gain dynamics $\Delta G\left(\lambda_{s}\right)$ due to the largest summation between absorption and emission cross-sections $\sigma_{e}^{\lambda_{s}}+$ $\sigma_{a}^{\lambda_{s}}$. This induced a maximum gain compression at the peak wavelength around $1534 \mathrm{~nm}$ as manifested in Fig. 3 . However, after this hump the gain exhibited modest ripple up to $1562 \mathrm{~nm}$.

Meanwhile, in contrast to the previous result an equal gain level was produced when incorporating two pump lasers at $977 \mathrm{~nm}$ (refer to Fig. 4). This was because the GEF was designed at $977 \mathrm{~nm}$ and the transmission spectra of GEF perfectly compensated the intended output profile over the whole wavelength region. The DGT was suppressed to the minimum value and the gain difference of $1.5 \mathrm{~dB}$ was observed 


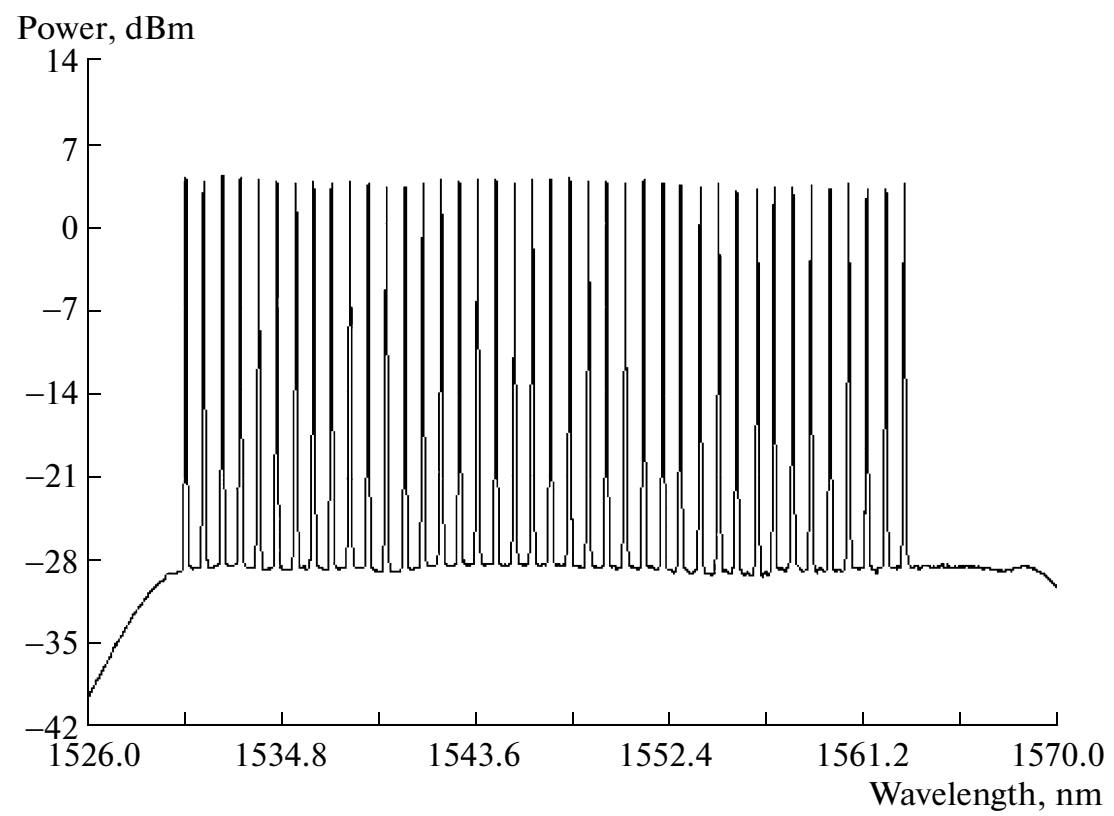

Fig. 4. Improved gain feature in the $\mathrm{C}$-band $(\mathrm{Gain}=30 \mathrm{~dB})$ in the setup that incorporated two infrared sources at $977 \mathrm{~nm}$.

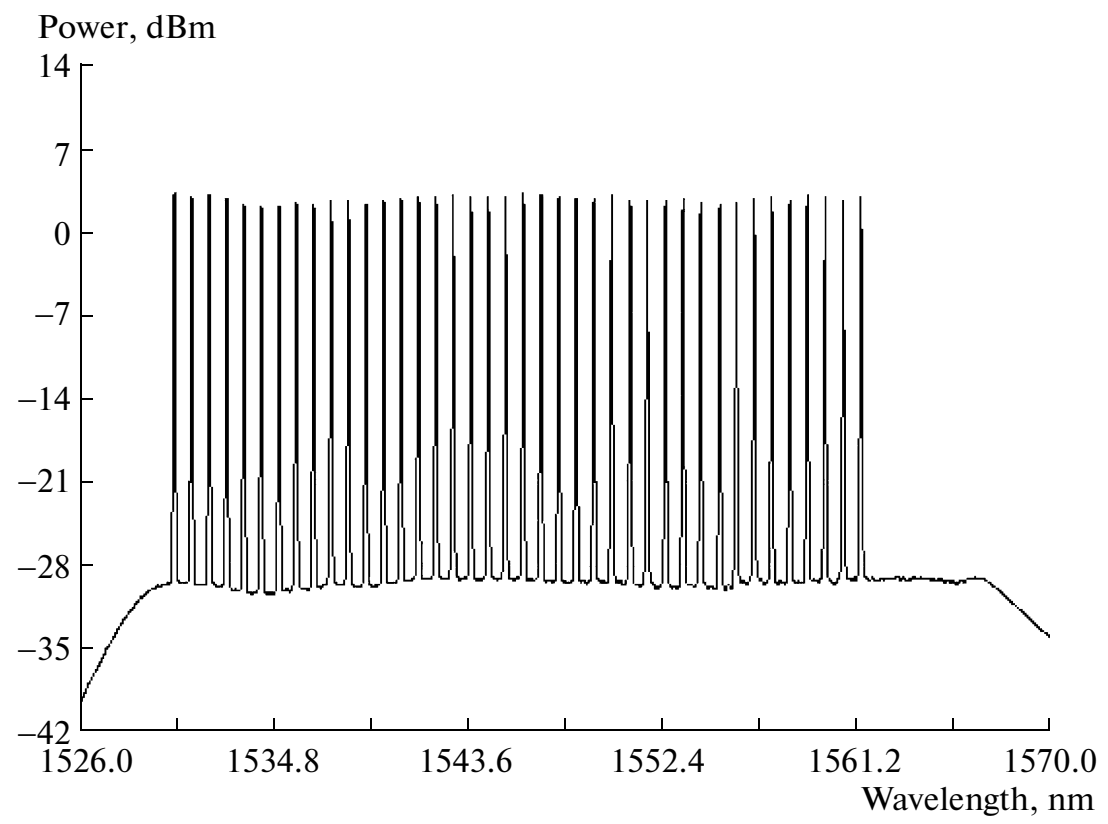

Fig. 5. Output spectrum from the amplifier module (Gain $=30 \mathrm{~dB}$ ) when implementing a $977 \mathrm{~nm}$ pump laser at $\mathrm{GB} \# 1$ and $\mathrm{GB} \# 3$ and a $980 \mathrm{~nm}$ pump source at GB\#2.

which was two times lower compared to that measured previously in Fig. 3. The uniformity in the gain operation was found to be analogous to those reported in prior works $[9,10]$.

Both of these outcomes from Figs. 3 and 4 are elucidated by the gain analysis in Eqs. (1) and (2). By relating the subjects to Fig. 2, the gain output features in this experiment were produced based on the proportionality between $\bar{n}_{2}$ and $\sigma_{a}^{\lambda_{p}}$. The spectral gain changes depended on $\bar{n}_{2}$ that was fixed at the designed pump wavelength of $977 \mathrm{~nm}$. When implementing $980 \mathrm{~nm}$ 
pump sources, the population inversion $\left(N_{2}-N_{1}\right)$ could not be controlled at the specific value that contributed to the flat gain spectrum. This was due to the significant difference in $\sigma_{a}^{\lambda_{p}}$ of about $0.32 \times 10^{-25} \mathrm{~m}^{2}$ within this $3 \mathrm{~nm}$ wavelength range and it is manifested by the distinctive dynamic gain tilt in Fig. 3. The remaining flat region in this figure represented a small gain discrepancy between two inversion factors at the designed one, $\bar{n}_{2}(977 \mathrm{~nm})$ and the experimental one, $\bar{n}_{2}(980 \mathrm{~nm})$. These clarify why the output spectrum was flatter at $977 \mathrm{~nm}$ (Fig. 4) compared to that in $980 \mathrm{~nm}$ (Fig. 3).

In the final assessment, in order to reduce the distinct ripple that was produced in Fig. 3, a combination of 977 with $980 \mathrm{~nm}$ pump sources was executed. The power characteristics were similar to the earlier experiments where the input power was $-7 \mathrm{dBm}$ and the output power was $23 \mathrm{dBm}($ Gain $=30 \mathrm{~dB})$. The result is depicted in Fig. 5 where a perfectly leveled spectral feature was observed for the whole C-band. The "hump" was suppressed at shorter wavelengths domain due to the effect of $\bar{n}_{2}$ at $977 \mathrm{~nm}$ and this transmission profile was acceptable for WDM applications. The gain difference was measured to be $1.6 \mathrm{~dB}$ which was comparable to that attained in Fig. 4. This result indicates that the combined pump wavelengths near-infrared sources could be utilized to suppress the fluctuation from the output gain of the EDFA module. In addition, the inclusion of a $980 \mathrm{~nm}$ pump laser was also beneficial in an amplifier system because of its efficiency to produce low-noise characteristics.

\section{CONCLUSIONS}

We have demonstrated successfully a spectral-independent gain operation from a four-stage EDFA module that was feasible for WDM applications. The gain equalizing filter was designed for the inversion population factor at $977 \mathrm{~nm}$. The pumping scheme was provided at wavelengths of 977 and $980 \mathrm{~nm}$ and it was implemented in three configurations. This pumping range implied a narrow spectroscopic bandwidth, but having a huge different of absorption cross-section around $0.32 \times 10^{-25} \mathrm{~m}^{2}$ that could initiate a considerable dynamic gain tilt. However, this was compensated by combining together a 977 and a $980 \mathrm{~nm}$ lasers in the pumping arrangement. From the measurement, the results indicate that gain consistencies were improved from 3.0 to $1.6 \mathrm{~dB}$ errors when employing appropriate integration of $980 \mathrm{~nm}$ band sources. This confirms the relationship between the inversion factor with the pump wavelength dependency that must be considered when designing and developing a gain-flattened EDFA.

\section{REFERENCES}

1. K.-H. Lin and J.-H. Lin, Laser Phys. Let. 5, 449 (2008).

2. M. A. Mahdi, A. K. Zamzuri, A. Ahmad, M. I. Md. Ali, and R. Mohamad, Laser Phys. Lett. 5, 296 (2008).

3. S.-K. Liaw, C.-K. Huang, and Y.-L. Hsiao, Laser Phys. Lett. 5, 543 (2008).

4. S. W. Harun, K. Dimyati, K. K. Jayapalan, and H. Ahmad, Laser Phys. Lett. 4, 10 (2007).

5. A. W. Naji, M. S. Z. Abidin, M. H. Al-Mansoori, A. R. Faidz, and M. A. Mahdi, Laser Phys. Lett. 4, 145 (2007).

6. C. H. Yeh, T. T. Huang, M. C. Lin, C. W. Chow, and S. Chi, Laser Phys. 19, 1246 (2009).

7. S. W. Harun, F. Abd Rahman, K. Dimyati, and H. Ahmad, Laser Phys. Lett. 3, 536 (2006).

8. P. F. Wysocki, J. Judkins, R. Espindola, M. Andrejco, A. Vengsarkar, and K. Walker, IEEE Photon. Technol. Lett. 9, 1343 (1997).

9. N. Kumar, M. R. Shenoy, and B. P. Pal, IEEE Photon. Technol. Lett. 17, 2056 (2005).

10. R. K. Vashney, B. Nagaraju, A. Singh, B. P. Pal, and A. K. Kar, Opt. Express 15, 13519 (2007).

11. M. H. Abu Bakar, M. A. Mahdi, M. Mokhtar, A. F. Abas, and N. Md. Yusof, Laser Phys. Lett. 6, 602 (2009).

12. H. Masuda and Y. Miyamoto, Electron. Lett. 44, 1082 (2008).

13. C. R. Giles, and D. Di Giovanni, IEEE Photon. Technol. Lett. 2, 797 (1990).

14. M. J. Yadlowsky, IEEE Photon. Technol. Lett. 11, 539 (1999).

15. C. R. Giles, and E. Desurvire, J. Lightwave Technol. 9, 271 (1991).

16. E. Desurvire, J. W. Sulhov, J. L. Zyskind, and J. R. Simpson, IEEE Photon. Technol. Lett. 2, 653 (1990). 